\title{
Boosted Three-Dimensional Black-Hole Evolutions with Singularity Excision
}

\author{
G. B. Cook, ${ }^{1}$ M. F. Huq, ${ }^{2}$ S. A. Klasky, ${ }^{3}$ M. A. Scheel, ${ }^{1}$ A. M. Abrahams, ${ }^{4,5}$ A. Anderson, ${ }^{6}$ P. Anninos,${ }^{4}$ \\ T. W. Baumgarte, ${ }^{4}$ N. T. Bishop, ${ }^{7}$ S. R. Brandt,${ }^{4}$ J. C. Browne, ${ }^{2}$ K. Camarda, ${ }^{8}$ M. W. Choptuik, ${ }^{2}$ R. R. Correll, ${ }^{2,9}$ \\ C. R. Evans, ${ }^{6}$ L. S. Finn, ${ }^{10}$ G. C. Fox,${ }^{3}$ R. Gómez,${ }^{11}$ T. Haupt,${ }^{3}$ L. E. Kidder, ${ }^{10}$ P. Laguna,${ }^{8}$ W. Landry,${ }^{1}$ L. Lehner, ${ }^{11}$ \\ J. Lenaghan, ${ }^{6}$ R. L. Marsa, ${ }^{2}$ J. Masso, ${ }^{4}$ R. A. Matzner, ${ }^{2}$ S. Mitra, ${ }^{2}$ P. Papadopoulos, ${ }^{8}$ M. Parashar, ${ }^{2}$ L. Rezzolla, ${ }^{4}$ \\ M. E. Rupright, ${ }^{6}$ F. Saied, ${ }^{4}$ P.E. Saylor, ${ }^{4}$ E. Seidel,${ }^{4}$ S. L. Shapiro, ${ }^{4}$ D. Shoemaker, ${ }^{2}$ L. Smarr,${ }^{4}$ W. M. Suen, ${ }^{12}$ \\ B. Szilágyi, ${ }^{11}$ S. A. Teukolsky, ${ }^{1}$ M. H. P. M. van Putten, ${ }^{1}$ P. Walker, ${ }^{4}$ J. Winicour, ${ }^{11}$ and J. W. York, Jr. ${ }^{6}$
}

(Binary Black Hole Grand Challenge Alliance)

\author{
${ }^{1}$ Cornell University, Ithaca, New York 14853 \\ ${ }^{2}$ The University of Texas at Austin, Austin, Texas 78712 \\ ${ }^{3}$ Syracuse University, Syracuse, New York 13244-4100 \\ ${ }^{4}$ University of Illinois at Urbana-Champaign, Urbana, Illinois 61801 \\ ${ }^{5}$ J. P. Morgan, 60 Wall St., New York, New York 10260 \\ ${ }^{6}$ University of North Carolina, Chapel Hill, North Carolina 27599 \\ ${ }^{7}$ University of South Africa, P.O. Box 392, Pretoria 0001, South Africa \\ ${ }^{8}$ Penn State University, University Park, Pennsylvania 16802 \\ ${ }^{9}$ Air Force Space Command, Peterson Air Force Base, Colorado 80914-4610 \\ ${ }^{10}$ Northwestern University, Evanston, Illinois 60208 \\ ${ }^{11}$ University of Pittsburgh, Pittsburgh, Pennsylvania 15260 \\ ${ }^{12}$ Washington University, St. Louis, Missouri 63130
}

(Received 26 November 1997)

\begin{abstract}
Binary black-hole interactions provide potentially the strongest source of gravitational radiation for detectors currently under development. We present some results from the Binary Black Hole Grand Challenge Alliance three-dimensional Cauchy evolution module. These constitute essential steps towards modeling such interactions and predicting gravitational radiation waveforms. We report on single black-hole evolutions and the first successful demonstration of a black hole moving freely through a three-dimensional computational grid via a Cauchy evolution: a hole moving near $6 M$ at $0.1 c$ during a total evolution of duration near 60M. [S0031-9007(98)05652-X]
\end{abstract}

PACS numbers: 04.70.Bw, 04.25.Dm, 04.30.Db

The accurate computational modeling of black-hole interactions is essential to the confident detection of astrophysical gravitational radiation by future space-based detectors such as LISA and by the LIGO/VIRGO/GEO complex of ground-based detectors currently under construction. The sensitivity of these detectors will be significantly enhanced if accurate computer simulations of black-hole mergers can produce predictions of radiation waveforms [1]. The Binary Black Hole Grand Challenge Alliance [2] was funded in September 1993 to develop the computational infrastructure for accurate simulations of the coalescence of black-hole binaries. The primary objective of the resulting code will be the prediction of waveforms from binary black-hole mergers. In this Letter we report on an important step towards achieving such simulations.

A key difficulty in evolving black-hole spacetimes is handling the curvature singularity contained within each hole. The only viable means of accomplishing this over time scales required for binary coalescence appears to be black-hole excision: exclude all or part of the blackhole interior (and the singularity) from the computational domain and evolve only the exterior region $[3,4]$. This is possible because, by definition, the region inside the hole cannot causally affect the exterior evolution. Black-hole excision has been implemented successfully in spherical symmetry by Seidel and Suen [5], Scheel et al. [6,7], Marsa and Choptuik [8], Anninos et al. [9], and Gómez et al. [10]; and in three dimensions (3D) by Daues [11], Anninos et al. [12], and by Gómez et al. [13].

We are developing a general algorithm to perform a Cauchy evolution of Einstein's equations on a domain containing black holes with excised interiors. Prior simulations of black-hole binaries have used coordinates in which the holes remain at fixed coordinate locations; for complicated motions, this will lead to undesirably contorted coordinates. In contrast, our more general approach allows black holes with excised interiors to move freely through the computational grid. Achieving this goal requires the synthesis of two key ingredients: a numerical scheme capable of stably evolving Einstein's equations on a domain containing moving boundaries (excised regions), and a set of gauge conditions that ensure that coordinates remain well behaved as black holes move through the grid. 
In this Letter we present a crucial step towards achieving a general black-hole evolution scheme: the first successful Cauchy computation of a single black hole freely moving through a 3D numerical grid. A different approach within the Alliance that uses a characteristic formulation [13] has recently shown 3D black-hole evolutions to $1400 M$ and success in moving a black hole less than one diameter.

Black-hole excision is based on the fundamental idea that the event horizon of a black hole is a natural causal boundary. Unfortunately, an event horizon cannot be located without knowing the full future evolution of the spacetime. However, a related structure known as an apparent horizon can usually be located on a spacelike time slice using only the information on that hypersurface. An apparent horizon is a topologically spherical spacelike two-surface on which the expansion of the congruence of outgoing null rays orthogonal to the surface vanishes. If the characteristic curves of all fields being evolved lie on or within the light cone, then the apparent horizon can be used as the inner boundary for a Cauchy evolution. The causal nature of this boundary implies that no explicit boundary condition need be imposed on the evolved quantities.

The gauge freedom (coordinate freedom) of general relativity allows considerable latitude in choosing how a computational solution evolves in time. Although gauge considerations cannot influence physics, they do determine how the coordinates and computational grid points used to describe the solution relate to physical locations in spacetime. A poor choice of spatial or temporal gauge can lead to coordinate pathologies that ruin a numerical simulation. For example, the proper distance between two adjacent computational grid points might approach zero or grow without bound. It is not fully understood what constitutes a good gauge choice.

An attractive choice $[8,14]$ for describing a single black hole is the ingoing Kerr-Schild form [15] of the Kerr metric:

$$
d s^{2}=g_{\mu \nu} d x^{\mu} d x^{\nu}=\left(\eta_{\mu \nu}+2 H l_{\mu} l_{\nu}\right) d x^{\mu} d x^{\nu},
$$
where $\mu, \nu$ run from 0 to $3, \eta_{\mu \nu}=\operatorname{diag}(-1,1,1,1), l^{\mu}$ is a null four-vector, and $H\left(x^{\alpha}\right)$ is a scalar function. In this gauge, the coordinates are closely related to the null structure of the spacetime. Furthermore, the solution is time independent (or has a trivial time dependence for a moving black hole), the spacelike hypersurfaces extend smoothly through the horizon, and gradients near the horizon are smaller than in several other coordinate choices.

Equation (1) is form invariant under Lorentz transformations, so it can be used to represent either a nontranslating or a boosted Kerr black hole. The $3+1$ decomposition of the spacetime metric leads to a metric $g_{i j}$ and extrinsic curvature $K_{i j}$ that we use as initial data and for comparisons at later times [16]. The lapse function,

and shift vector,

$$
\beta_{i}=2 H l_{t} l_{i}
$$

are analytic functions of space and time that we impose as gauge conditions. For the time-independent Schwarzschild spacetime, $H=M / r$ ( $M$ is the mass), $l_{\mu}=\left(1, x_{i} / r\right)$, and the apparent horizon coincides with the event horizon. In fact, the apparent and event horizons coincide in the boosted case as well.

Schemes for excising the interior of a black hole from the computational grid typically require a superluminal shift vector in some region of the computational domain, and must cope with the lack of an explicit boundary condition on the excision boundary. The Alliance Cauchy evolution module implements an evolution scheme that is designed to provide a stable evolution for any choice of shift vector. A typical evolution equation has the form

$$
\left(\partial_{t}-£_{\beta}\right) T=\ldots,
$$

where $\mathfrak{f}_{\beta}$ is the Lie derivative along the shift vector and $T$ is an arbitrary spatial tensor. We can rewrite this as

$$
\partial_{0} T-\left(\mathfrak{f}_{\beta}-\beta^{i} \partial_{i}\right) T=\ldots,
$$

where $\partial_{0} \equiv \partial_{t}-\beta^{i} \partial_{i}$ is a time derivative in the direction normal to the spatial hypersurface and which, by definition, is centered in the light cone. This scheme removes from $\mathfrak{f}_{\beta}$ the advective term that potentially leads to evolution along a nontimelike direction. This guarantees that a numerical evolution will be stable against instabilities produced by superluminal shifts. Note that the tensor components being evolved remain in the coordinate basis $\partial / \partial x^{i}$ that is Lie dragged along the $t^{\mu}$ direction, but the computational grid points at which these components are defined do not remain at constant values of $x^{i}$. Instead, the grid points remain at constant values of the spatial coordinates $\tilde{x}^{i}$ that are Lie dragged along the unit normal to the spatial hypersurface. The evolved quantities are determined at the desired spatial coordinate locations $x^{i}$ by interpolation. This interpolation requires that the $\tilde{x}^{i}$ coordinate values be evolved along the $t^{\mu}$ direction for each point where the evolved quantities are to be evaluated on a given time slice. It also obviates the need for a boundary condition at the excision surface. The algorithm can easily accommodate black holes that move through the grid. In this case, unused grid points that had been in the interior of a hole can move into the exterior. These new points will be filled by the interpolation phase and will always be filled from data that are in the future domain of dependence of the previous time slice. Similar schemes were developed by Seidel and Suen [5], Alcubierre and Schutz [17], and Daues [11]. Additional details on how this evolution scheme is implemented can be found in Scheel et al. [7] and in a forthcoming paper [18] that will describe the details of the Alliance Cauchy evolution module. The key point is that by splitting the evolution along $t^{\mu}$ into an evolution along the unit normal followed by an interpolation, the algorithm guarantees that the numerical 
evolution step is always taken in the center of the physical light cone.

The Cauchy evolution module developed by the Alliance is based on the standard $(3+1)$, or ADM, decomposition of Einstein's field equations [19,20]. The module uses Cartesian coordinates and evolves the threemetric $g_{i j}$, and extrinsic curvature $K_{i j}$, on a 3D rectangular grid. The evolution equations are solved using an iterative Crank-Nicholson differencing scheme. The constraint equations are not imposed on the solution but are utilized as a diagnostic.

We present results from two sets of black-hole experiments: evolutions of nontranslating and of boosted Schwarzschild black holes. In both sets of experiments we work with a 3D $n \times n \times m$ computational grid (where the grid spacing is uniform and equal in all directions), and we impose Dirichlet boundary conditions on the grid faces throughout the evolutions. For the nontranslating cases, this means freezing the outer boundaries to initial data. For the boosted case, this means resetting the exact solution at the faces for each time step. At each time during the course of our evolution we impose the exact gauge conditions (2) and (3) as a function of four spacetime coordinates. We allow for a "buffer region" by placing the inner boundary (excised region) a short distance inside the apparent horizon (where the inner boundary might have been placed). This distance is set to $p$ times the grid spacing.

For the case of a boosted black hole we discuss two runs; one with $p=3$ buffer zones and the other with $p=5$ buffer zones between the horizon and the inner boundary. Both runs use $n=33$ and $m=65$ with a domain of $-(8 / 3) M$ to $8 M$ in the $z$ direction and a domain of

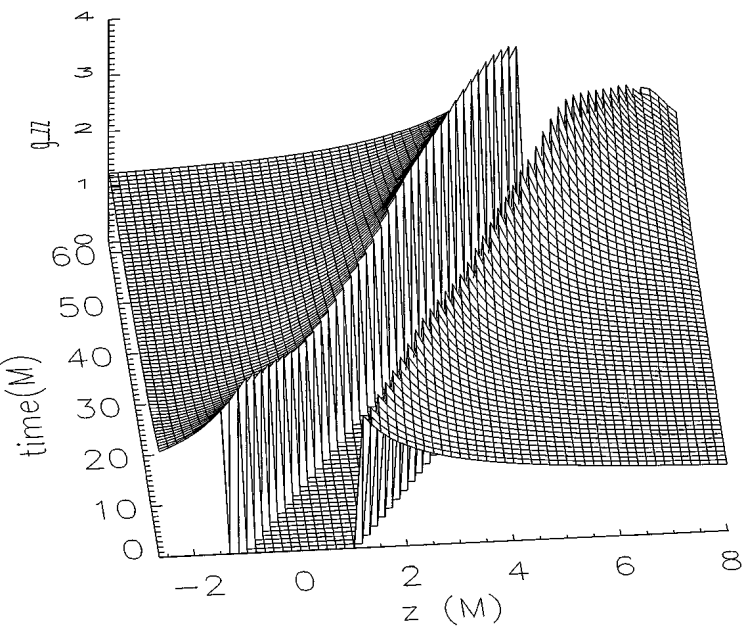

FIG. 1. Metric component $g_{z z}$ along the $z$ axis as a function of time. The flat region that moves diagonally to the right represents the excised region (inside the black hole). Note that points at the trailing edge (left side) are smoothly updated as the hole moves towards positive $z$. Coordinate effects are seen to appear near the inner boundary.
$-(8 / 3) M$ to $(8 / 3) M$ in the $x$ and $y$ directions. The black hole is initially located at the origin and has a velocity $v=0.1 c$ in the $z$ direction. The evolution was carried out with a Courant factor of $1 / 4$. Figure 1 shows a spacetime picture of $g_{z z}$ along the $z$ axis vs time for the $p=5$ run. In this case we evolved the black hole for $61 M$, moving three hole radii. The evolution terminated when the leading edge of the black hole was within five gridpoints of the $z=$ $8 M$ face. At this point the differencing algorithms failed because of an insufficient number of points between the inner and outer boundaries. In the $p=3$ run the black hole evolved for $54 \mathrm{M}$ with coordinate stretching occurring at the trailing edge. We find that the addition of buffer zones enhanced the run and reduced coordinate stretching for the duration of the $p=5$ run. This is consistent with the behavior of the nontranslating runs described below. As the hole moved in each of the evolutions, many coordinate points emerged stably and smoothly from the excised region into the computational domain. Figure 2 shows a spacetime picture of the normalized Hamiltonian constraint diagnostic along the $z$ axis for the $p=5$ run. Note that the Hamiltonian constraint is well behaved in the regions where grid points have emerged from the black hole.

For the case of a nontranslating black hole, we chose $n=m=49$. The outer boundaries are placed at $-4 M$ and $4 M$ in each direction. With a Courant factor of $1 / 4$ we can evolve a single black hole to $95 M$. This is an encouraging achievement comparable to the longest 3D single black-hole Cauchy simulation ( $\sim 138 M$; Daues [11] and private communication).

As in the moving hole case, the length of the evolutions is dependent on the placement of the inner boundary. We found that by setting the number of buffer zones to $p=0,1,5$, and 9 , we can run a nonmoving hole to a maximum time of $t_{\max }=16 M, 20 M, 95 M$, and $82 M$. In all cases, the evolution terminates because the iterative Crank-Nicholson evolution scheme fails to

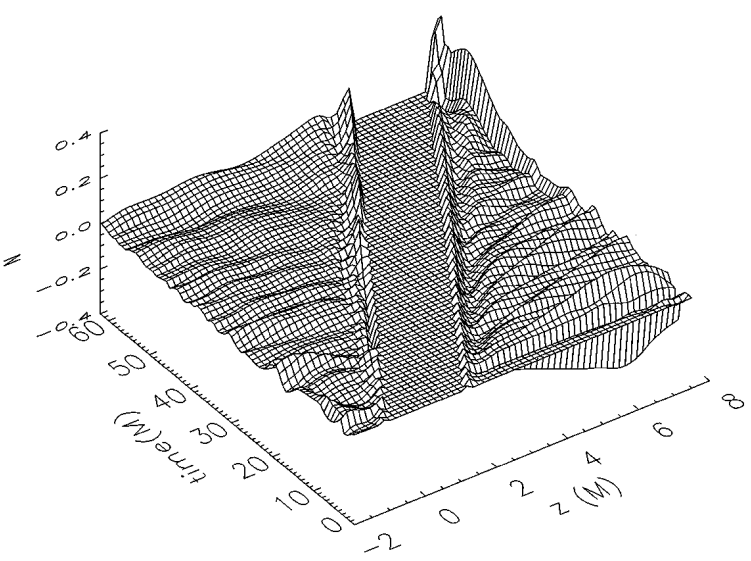

FIG. 2. Normalized Hamiltonian constraint, $N=\left(R+K^{2}-\right.$ $\left.K^{i j} K_{i j}\right) /\left(|R|+\left|K^{2}\right|+\left|K^{i j} K_{i j}\right|\right)$, along the $z$ axis as a function of time. The flat region shows the location of the excised part of the domain within the hole. 
converge to a solution. While the evolved solutions all deviated from the analytic solution, the behavior in each case was somewhat different and a definitive explanation of why each evolution fails requires further testing. The most likely causes of the late time problems are either coordinate effects and/or numerical instabilities due to choices of finite difference operators. One such coordinate effect can come from fixing the gauge via the analytic functions (2) and (3). As numerical evolutions progress, the evolved data drift from the exact solution. When this happens, the choice of lapse and shift being used will no longer enforce the desired, underlying KerrSchild gauge condition. In this case, effects such as grid stretching can occur and the coordinate location of the apparent horizon will no longer coincide with the analytic solution. For $p=0$ or 1 , the evolution progresses rather smoothly and the computed location of the apparent horizon gradually moves inward. Eventually the apparent horizon passes within the computational inner boundary, so that the inner boundary becomes timelike and therefore unsuitable as a boundary for black-hole excision. The evolutions terminate soon after this occurs. For $p=5$ or 9, the evolution is more complicated. The relatively smooth growth in error with the apparent horizon moving inward no longer occurs (or occurs on a much longer time scale). In these cases, the dominant errors appear to be noise introduced at the Dirichlet outer boundaries. These errors propagate across the computational grid and appear to be amplified in some way. It is possible that the analytic gauge conditions are causing this amplification; some unusual coupling with the inner boundary may also be responsible. Eventually, the geometry near the inner boundary becomes quite nonspherical. While we are no longer certain of the location of the apparent horizon at this point, we believe that the inner boundary again becomes timelike prior to the evolution ending.

The fact that the evolutions depend strongly on the placement of the inner boundary agrees with prior 3D work by Daues [11] but requires further study. Using a larger number of buffer zones forces the inner boundary deeper into the black-hole interior where an increasingly larger propagation speed is required for information to escape from the black hole. Thus the strong dependence of the evolutions on $p$ could indicate some nonphysical gauge-dependent quantities or numerical errors propagating faster than light. Resolution of this question must await at least a consistent set of convergence tests to understand the effect of $p$ as the discretization is refined. In spite of these concerns, the evolution to $95 M$ is an encouraging result, and we believe that improved gauge choices that utilize information from the evolution (e.g., the computed apparent horizon location, cf. Refs. [7,11]) will allow for much longer evolutions.

In the evolutions of nontranslating holes, each case above results in an unstable evolution. We believe that this is most likely due to coordinate effects. This belief is supported by evolving a region adjacent to a nontranslating black hole. We consider a domain extending from $-(3 / 2) M$ to $(3 / 2) M$ in all directions and displace the center of the black hole along one axis by $2 M$. By this design the horizon passes through the center of the computational cube. Using resolution $n=m=49$ and Dirichlet boundary conditions on all boundaries, we find that the solution settles down to a steady state because of numerical dissipation and perhaps because wave disturbances fall within the horizon. At low enough resolution, the errors in the analytic gauge conditions and Dirichlet boundaries were too large to allow a steady-state solution. As a rule, higher resolution cannot force a fundamentally unstable numerical scheme to be "more stable." We thus find this test to be highly suggestive that our interior evolution scheme is fundamentally stable and our principal problems are with coordinate and boundary effects.

All of the above results are very encouraging for the ultimate goal of evolving multiple black-hole spacetimes. In both the boosted and nontranslating evolutions, improvements to the coordinate conditions are clearly necessary. Nonanalytical coordinate conditions need to be developed for the boosted case and we are hopeful that it will be possible to formulate a general Kerr-Schild-like gauge condition that does not require the solution of elliptic equations. Other combinations of spatial and temporal gauge conditions may also work or may even be necessary. There is still considerable work to be done on the computational infrastructure required to support general binary black-hole simulations, but it is clear that one of the keys to successful evolutions is understanding which gauge conditions are appropriate for multiple black holes.

This work was supported by the NSF Binary Black Hole Grand Challenge Grants No. NSF PHY/ASC 9318152 (ARPA supplemented), No. NSF PHY 93-10083, and Metacenter Grant No. MCA94P015N. Computations were performed at NPAC (Syracuse University), at NCSA (University of Illinois at Urbana-Champaign), and at the Pittsburgh Supercomputing Center.

[1] É.É. Flanagan and S.W. Hughes, Report No. gr-qc/ 9701039 (to be published).

[2] http://www.npac.syr.edu/projects/bh/

[3] J. Thornburg, Classical Quantum Gravity 4, 1119 (1987).

[4] W. Unruh (private communication).

[5] E. Seidel and W.-M. Suen, Phys. Rev. Lett. 69, 1845 (1992).

[6] M. A. Scheel, S. L. Shapiro, and S. A. Teukolsky, Phys. Rev. D 51, 4208 (1995).

[7] M. A. Scheel et al., Phys. Rev. D 56, 6320 (1997).

[8] R. L. Marsa and M. W. Choptuik, Phys. Rev. D 54, 4929 (1996).

[9] P. Anninos et al., Phys. Rev. D 51, 5562 (1995).

[10] R. Gómez, R. L. Marsa, and J. Winicour, Phys. Rev. D 56, 6310 (1997). 
[11] G. Daues, Ph.D. thesis, Washington University, 1996 (unpublished).

[12] P. Anninos et al., Phys. Rev. D 52, 2059-2082 (1995).

[13] R. Gómez, L. Lehner, R. L. Marsa, and J. Winicour, Report No. gr-qc/9710138.

[14] G. Allen et al., in Proceedings of the Seventh Marcel Grossmann Meeting on General Relativity, edited by R. T. Jantzen and G. M. Keiser (World Scientific, Singapore, 1996), pp. 615-618.

[15] C. W. Misner, K.S. Thorne, and J.A. Wheeler, Gravitation (W.H. Freeman and Company, New York, New York, 1973).
[16] M.F. Huq, Ph.D thesis, The University of Texas at Austin, 1996 (unpublished).

[17] M. Alcubierre and B. F. Schutz, J. Comput. Phys. 112, 44 (1994).

[18] G. B. Cook, M. F. Huq, S. A. Klasky, and M. A. Scheel (to be published).

[19] R. Arnowitt, S. Deser, and C. W. Misner, in Gravitation: An Introduction to Current Research, edited by L. Witten (John Wiley and Sons, New York, 1962), pp. 227-265.

[20] J. W. York, Jr., in Sources of Gravitational Radiation, edited by L.L. Smarr (Cambridge University Press, Cambridge, England, 1979), pp. 83-126. 